\title{
An Epidemiological Simulation Model with Three Scales of Spatial Hierarchy
}

\author{
Laetitia Willocquet and Serge Savary
}

First author: UMR INRA-ENSAR BiO3P (Biologie des Organismes et des Populations appliquée à la Protection des Plantes), BP 35327, 35653 Le Rheu Cedex, France; and second author: Ecole Nationale Supérieure Agronomique de Rennes, 65 rue de Saint-Brieuc, CS 84215, 35042 Rennes Cedex, France. Accepted for publication 5 April 2004.

\begin{abstract}
Willocquet, L., and Savary, S. 2004. An epidemiological simulation model with three scales of spatial hierarchy. Phytopathology 94:883-891.

An epidemiological model integrating three organizational scales of host plant populations (e.g., sites, leaves, and plants) is presented. At the lowest (site) scale, the model simulates the dynamics of vacant, latent, infectious, and removed sites. Three types of vacant sites are distinguished, depending on presence of infections at higher scales (leaf or plant). The rate of infection of each type of vacant site is computed according to ratios of autodeposition, allo-leaf-deposition, and allo-plantdeposition. At the leaf and plant scales, the rate of victimization is a function of the rate of infection of vacant sites. Sensitivity analyses showed

outputs conformed with results from other approaches in the case of random distribution of the disease. The model hypotheses concerning infection from autodeposited propagules, and their implications for disease epidemics, are discussed. The model can be used to derive relationships between allo-deposition ratios and disease incidences at the three scales. These relationships become simple when disease intensity is low. These relationships may be useful, e.g., to assess the potential efficiency of cultivar mixture to control epidemics. Integration of different organization scales and allo-deposition parameters enables the model to capture important features of epidemics developing in space without using explicitly spatialized variables. Such an approach could be useful to analyze other ecological processes that involve a variety of scales.
\end{abstract} that deposition patterns (the relative proportions of auto-, allo-leaf-, and allo-plant-depositions) and host structure (leaf size and number of leaves per plant) affected the speed of epidemics at the different scales. Model
Additional keywords: canopy structure, disease aggregation.
The early epidemiological models to represent the dynamics of plant disease epidemics, e.g., the generic models developed by Van der Plank (30) and Zadoks (38), were developed under the hypothesis of random disease distribution. In spite of this very stringent hypothesis, these models successfully represented a wide range of plant disease epidemics, with varying spatial structures. An explanation of this paradox was proposed by Waggoner and Rich (31), who incorporated a parameter for disease aggregation in a logistic model, thus implicitly accounting for the spatial distribution of disease. Their representation of disease aggregation involved the parameter $k$ of the negative binomial distribution. $k$ varies from zero to infinity and decreases as aggregation increases. Waggoner and Rich (31) also showed that their model resolved to a logistic one when $k$ equaled 1 , and explained in this way why contagious diseases could develop according to a logistic law. Other epidemiological models have implicitly integrated spatial heterogeneity by modifying the correction factor (COFR; $38)$, and applied this approach to specific pathosystems $(21,36,37)$.

However, different approaches have been developed, where the one- $(15,16)$ or two-dimensional structures $(4,22,28,34,39,40)$ of plant disease epidemics were explicitly represented. An important attribute of these models is the contact distribution (28), that is, the probability of a pathogen propagule encountering a host site, or the distribution of dispersed spores, in the case of aerially dispersed pathogens. The contact distribution depends on the distance between the source (where propagules are emitted) and the target site (where propagules are deposited). It has been given dif-

Corresponding author: L. Willocquet; E-mail address: willocqu@ rennes.inra.fr

Publication no. P-2004-0617-03R

(C) 2004 The American Phytopathological Society ferent distribution shapes, including the two-dimensional Gaussian $(28,39)$, Bessel (29), double geometric (15), or half-Cauchy (34).

Spatial patterns can be addressed by considering changes in scales within nested units and subunits that represent different levels of organization of the host population. For example, one may consider a field as a population of plants, each consisting in a population of leaves, and each in turn, consisting in a population of leaflets, or sites. Statistical approaches have recently been developed to assess the relationships between disease intensity at different scales and disease aggregation $(8,14,26,27)$. The dispersal of propagules can be greatly affected by spatial hierarchies of the source and of the target. The probability of a propagule to be dispersed within the same plant may be greater than the probability of a propagule to be dispersed to another plant. These probabilities are also influenced by the structure of the host. Strong relationships between canopy structure and disease aggregation have been shown in rice sheath blight $(19,32)$ or strawberry leaf blight (26). Conversely, disease gradients are also affected by canopy structure in many epidemics $(5,12)$, including soybean aerial blight (35), barley powdery mildew (2), or groundnut late leaf spot (20).

The objective of the model presented here was to incorporate the spatial structure of an epidemic using auto- and allo-deposition ratios and to consider three scales in a spatial hierarchy: site, leaf, and plant. The model was then used to assess the effects of auto- and allo-deposition and host structure on epidemiological development.

\section{THEORY AND APPROACHES}

General structure. The three nested levels of hierarchy of the model (sites, leaves, and plants) are shown in Figure 1, and model variables are listed in Table 1 . The model simulates the infection cycle at the site scale, and leaf and plant victimization (sensu van den Bosch et al. [28]), with a 1-day time step. 
At the site scale, the model distinguishes three types of infections: (i) infection of sites located on victimized (diseased) leaves, (ii) infection of sites belonging to healthy leaves within a victimized plant, and (iii) infection of sites located on healthy plants. Distinguishing these three types of infection allows the incorporation of epidemiological parameters for disease spread at different scales, such as ratios of propagule autodeposition and allo-deposition. The terms propagule and spore are used interchangeably. Autodeposition is the deposition of a propagule produced on a lesion located on the same leaf. Two cases of allo-deposition are considered: allo-leaf-deposition, which is deposition of a propagule produced on a lesion located on a different leaf, but in the same plant; and allo-plant-deposition, which is deposition of a propagule produced on a lesion located on a different plant. The victimization of leaves is a function of site infection through alloleaf- and allo-plant-deposition. The victimization of plants depends on the infection of sites located on healthy plants.

Further assumptions of the model are as follows: the system is closed, with no propagules flowing outside, and no propagules entering the system; the number of sites, leaves, and plants is assumed constant over time; and lesion expansion is disregarded.

Infection at the site scale: General shape of the equation for infection rate. Three types of state variables for vacant sites (Fig. 1) are defined: VSIL, the number of vacant sites located on victimized (or diseased) leaves; VSIP, the number of vacant sites located on healthy leaves in victimized plants; and VSHP, the number of vacant sites located on healthy plants. All three types of sites, when infected, become latent (LSIL, LSIP, LSHP), merge into a single type of infectious site (INFSI), and finally become noninfectious (removed sites that cannot be infected anymore [REMSI]). As lesion expansion is not considered in the model, infected sites (latent, infectious, and removed) have the same size.

Each vacant site type can be infected with an infection rate $\left(\right.$ rinf $\left._{i}\right)$ that has the following general shape:

$$
\operatorname{rinf}_{i}(t)=\operatorname{INFSI}(t) \times \mathrm{dmfr} \times \mathrm{f}_{i}(t) \times \operatorname{cofr}_{i}(t)
$$

where $i=\mathrm{IL}$, IP, and HP, for the vacant sites VSIL, VSIP, and VSHP, respectively; and dmfr is the daily multiplication factor (sensu Zadoks [38]). One additional hypothesis is that dmfr is constant; $f_{i}$ is the proportion of propagules deposited on the corresponding type of vacant site, relative to the total number of propagules deposited; and $\operatorname{cofr}_{i}(t)$ is the proportion of sites available for infection for each type of vacant site.

Definition of $f_{i}$. Propagule dispersal is assumed to occur at one of the three levels of hierarchy, according to three ratios. The first ratio, auto (Table 1), is the autodeposition ratio, which is the proportion of deposited spores originating from lesions located on the same leaf. alloL is the allo-leaf-deposition ratio, which is the proportion of deposited spores originating from lesions located on a different leaf, in the same plant. alloP is the allo-plant-deposition ratio, which is the proportion of deposited spores originating from lesions located on a different plant. All three ratios are made relative to the total number of deposited spores, and their sum equals 1 .

$f_{\mathrm{HP}}(t)$ is the proportion of spores that are deposited on vacant sites located on healthy plants, relative to the total number of spores deposited. Spores deposited on healthy plants necessarily originate from a different plant, and thus concern allo-plant-deposition. Among spores involved in allo-plant-deposition, a proportion is deposited on diseased plants, and the remaining spores are deposited on healthy plants. It is assumed that the proportion of spores involved in allo-plant-deposition that are deposited on

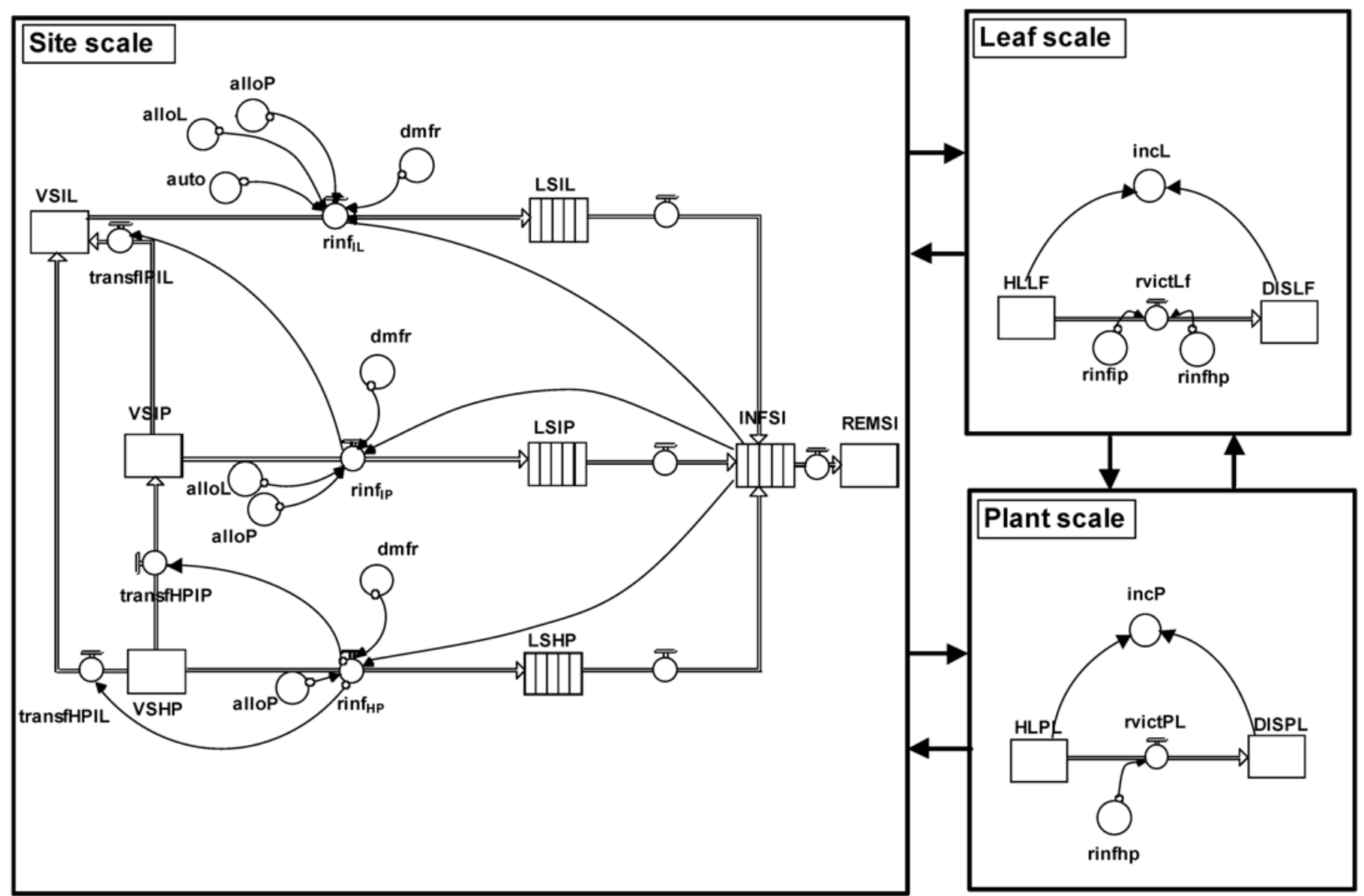

Fig. 1. Simplified structure of the three-scale simulation model incorporating autodeposition, allo-leaf-deposition, and allo-plant-deposition. Symbols of variables are listed in Table 1. 
healthy plants equals the proportion of healthy plants. The term $f_{\mathrm{HP}}(t)$ can thus be written as

$$
f_{\mathrm{HP}}(t)=\operatorname{alloP} \times[1-\operatorname{incP}(t)]
$$

where $\operatorname{incP}(t)$ is the plant incidence, which is the proportion of diseased plants.

$f_{\mathrm{IP}}(t)$ is the proportion of spores that are deposited on vacant sites located on healthy leaves in diseased plants, relative to the total number of spores deposited. This proportion is thus related to spores that are deposited through allo-plant-deposition and allo-leaf-deposition. Among spores deposited through allo-plantdeposition, only those deposited on healthy leaves of diseased plants correspond to VSIP sites. Also, among spores deposited through allo-leaf-deposition, only those deposited on healthy leaves of diseased plants correspond to VSIP sites. It is assumed that the proportion of spores deposited on healthy leaves of diseased plants is the proportion of healthy leaves in diseased plants. The term $f_{\mathrm{IP}}(t)$ can thus be written as

$$
f_{\mathrm{IP}}(t)=\{\operatorname{alloP} \times \operatorname{incP}(t) \times[1-\operatorname{cincL}(t)]\}+\{\operatorname{alloL} \times[1-\operatorname{cincL}(t)]\}
$$

where $\operatorname{cincL}(t)$ is the conditional (34) leaf incidence, which is the proportion of victimized leaves within the population of victimized plants.

$f_{\mathrm{IL}}(t)$ is the proportion of spores that are deposited on vacant sites located on diseased leaves in diseased plants, relative to the total number of spores deposited. This proportion is thus related to spores that are deposited through autodeposition, allo-leafdeposition, and allo-plant-deposition. By definition, spores deposited through autodeposition are deposited on VSIL sites only (the only type of healthy sites located on diseased leaves). Among spores deposited through allo-plant-deposition, only those deposited on diseased leaves of diseased plants correspond to VSIL sites. Also, among spores deposited through allo-leaf-deposition, only those deposited on diseased leaves of diseased plants correspond to VSIL sites. The term $f_{\mathrm{IL}}(t)$ can thus be written as

$$
f_{\mathrm{IL}}(t)=\text { auto }+[\text { alloP } \times \operatorname{incP}(t) \times \operatorname{cincL}(t)]+[\operatorname{alloL} \times \operatorname{cincL}(t)]
$$

From equations 2, 3, and 4, it can be derived that the sum of the three proportions of $f_{\mathrm{HP}}, f_{\mathrm{IL}}$, and $f_{\mathrm{IP}}$ equals one, reflecting the three-level structure of the model.

Definition of $\operatorname{cofr}_{i} \cdot \operatorname{cofr}_{\mathrm{HP}}$ is the proportion of VSHP sites available for infection. In the case of VSHP (vacant sites on healthy plants), cofr ${ }_{\mathrm{HP}}$ equals one, because VSHP are vacant sites on healthy plants.

Similarly, cofr $\mathrm{IP}_{\mathrm{IP}}$ is the proportion of sites on healthy leaves in diseased plants that are still vacant. cofr ${ }_{I P}$ also equals one, because VSIP are vacant sites on healthy leaves.

$\operatorname{cofr}_{\mathrm{IL}}$, the proportion of sites located on diseased leaves that are still vacant, can be written as

$$
\operatorname{cofr}_{\text {IL }}(t)=1-\operatorname{cincS}(t)
$$

where $\operatorname{cincS}(t)$ is the conditional site incidence, i.e., the proportion of infected sites within the population of diseased leaves.

Equations for infection of the three types of vacant sites. From equations 1 to 5, the rates of infection for each type of vacant site can be written as

$$
\begin{gathered}
\operatorname{rinf}_{\mathrm{IL}}(t)=\operatorname{INFSI}(t) \times \operatorname{dmfr} \times\{\text { auto }+[\operatorname{alloP} \times \operatorname{incP}(t) \times \operatorname{cincL}(t)]+ \\
[\text { alloL } \times \operatorname{cincL}(t)]\} \times[1-\operatorname{cincS}(t)] \\
\operatorname{rinf}_{\mathrm{IP}}(t)=\operatorname{INFSI}(t) \times \operatorname{dmfr} \\
\times(\{\text { alloP } \times \operatorname{incP}(t) \times[1-\operatorname{cincL}(t)]\}+\{\text { alloL } \\
\times[1-\operatorname{cincL}(t)]\}) \\
\operatorname{rinf}_{\mathrm{HP}}(t)=\operatorname{INFSI}(t) \times \operatorname{dmfr} \times \operatorname{alloP} \times[1-\operatorname{incP}(t)]
\end{gathered}
$$

Transfer of vacant sites. When a vacant site of type VSIP becomes infected (Fig. 1), this site flows toward the latent state LSIP. Simultaneously, all other vacant sites belonging to the same leaf are transferred to the category of vacant sites belonging to diseased leaves (VSIL-type sites). The rate of transfer of vacant sites from VSIP to VSIL can be written as

$$
\operatorname{transfIPIL}(t)=\operatorname{rinf}_{\mathrm{IP}}(t) \times(\text { nsiperLf }-1)
$$

where nsiperLf is the number of sites per leaf. The underlying assumption of equation 9 is that, at each time step, propagules involved in allo-leaf- and allo-plant-deposition that are deposited on healthy leaves are deposited on separate ones.

When a vacant site of type VSHP becomes infected (Fig. 1), this site flows toward the latent state LSHP (latent site on an otherwise healthy plant). Similar to events following the infection of a VSIP site, other vacant sites of the same leaf become VSIL. Furthermore, all other vacant sites belonging to the other healthy leaves of the plant become VSIP. The rates of transfer of vacant

\begin{tabular}{|c|c|c|}
\hline Acronym & Dimension & Meaning \\
\hline alloL & $\ldots$ & $\begin{array}{l}\text { Allo-leaf-deposition ratio (inter-leaf, } \\
\text { intra-plant) }\end{array}$ \\
\hline alloP & $\ldots$ & Allo-plant-deposition ratio (inter-plant) \\
\hline auto & $\ldots$ & Auto-deposition ratio (intra-leaf) \\
\hline cincL & $\ldots$ & $\begin{array}{r}\text { Conditional leaf incidence (proportion of } \\
\text { diseased leaves within diseased plants) }\end{array}$ \\
\hline $\operatorname{cincS}$ & $\ldots$ & $\begin{array}{l}\text { Conditional site incidence (proportion of } \\
\text { infected sites within diseased leaves) }\end{array}$ \\
\hline DISLF & Nleaf & Number of diseased leaves \\
\hline DISPL & Nplant & Number of diseased plants \\
\hline $\mathrm{dmfr}$ & Nsites Nsites ${ }^{-1}$ day $^{-1}$ & Daily multiplication factor \\
\hline HLLF & Nleaf & Number of healthy leaves \\
\hline HLPL & Nplant & Number of healthy plants \\
\hline incL & $\ldots$ & $\begin{array}{l}\text { Leaf incidence (proportion of diseased } \\
\text { leaves) }\end{array}$ \\
\hline incP & ... & $\begin{array}{l}\text { Plant incidence (proportion of diseased } \\
\text { plants) }\end{array}$ \\
\hline INFSI & Nsites & Number of infectious sites \\
\hline LSHP & Nsites & $\begin{array}{l}\text { Number of latent sites that were located on } \\
\text { a healthy plant at the time of infection }\end{array}$ \\
\hline LSIL & Nsites & $\begin{array}{l}\text { Number of latent sites that were located on } \\
\text { a diseased leaf at the time of infection }\end{array}$ \\
\hline LSIP & Nsites & $\begin{array}{l}\text { Number of latent sites that were located } \\
\text { on a healthy leaf within a diseased } \\
\text { plant at the time of infection }\end{array}$ \\
\hline nsiperLf & Nsites Nleaf ${ }^{-1}$ & Number of sites per leaf \\
\hline nsiperPl & Nsites Nplant ${ }^{-1}$ & Number of sites per plant \\
\hline REMSI & Nsites & Number of removed sites \\
\hline $\operatorname{rinf}_{\mathrm{HP}}$ & Nsites day $^{-1}$ & Rate of infection of VSHP sites \\
\hline $\operatorname{rinf}_{I L}$ & Nsites day $^{-1}$ & Rate of infection of VSIL sites \\
\hline $\operatorname{rinf}_{I P}$ & Nsites day $^{-1}$ & Rate of infection of VSIP sites \\
\hline rvictLF & Nleaf day ${ }^{-1}$ & Rate of leaf victimization \\
\hline rvictPL & Nplant day $^{-1}$ & Rate of plant victimization \\
\hline transfHPIL & Nsites day $^{-1}$ & $\begin{array}{l}\text { Rate of transfer of VSHP sites toward } \\
\text { VSIL sites }\end{array}$ \\
\hline transfHPIP & Nsites day $^{-1}$ & $\begin{array}{l}\text { Rate of transfer of VSHP sites toward } \\
\text { VSIP sites }\end{array}$ \\
\hline transfIPIL & Nsites day $^{-1}$ & $\begin{array}{l}\text { Rate of transfer of VSIP sites toward } \\
\text { VSIL sites }\end{array}$ \\
\hline VSHP & Nsites & $\begin{array}{l}\text { Number of vacant sites located on a } \\
\text { healthy plant }\end{array}$ \\
\hline VSIL & Nsites & $\begin{array}{l}\text { Number of vacant sites located on a } \\
\text { diseased leaf }\end{array}$ \\
\hline VSIP & Nsites & $\begin{array}{l}\text { Number of vacant sites located on a } \\
\text { healthy leaf within a diseased plant }\end{array}$ \\
\hline
\end{tabular}
sites from VSHP to VSIL and VSIP (Fig. 1) are written as

$$
\operatorname{transfHPIL}(t)=\operatorname{rinf}_{\mathrm{HP}}(t) \times(\text { nsiperLf }-1)
$$

$$
\operatorname{transfHPIP}(t)=\operatorname{rinf}_{\mathrm{HP}}(t) \times(\text { nsiperPl }- \text { nsiperLf })
$$

where nsiperPl is the number of sites per plant. Again, equations 10 and 11 assume that propagules involved in allo-plant-deposition and dispersed on healthy plants are deposited on separate ones.

TABLE 1 . List of the variables used in the model 
Leaf and plant victimization. The rate of plant victimization (plants becoming diseased, Fig. 1) corresponds to the rate of infection of healthy sites located on healthy plants (VSHP sites) and equals $\operatorname{rinf}_{\mathrm{HP}}(t)$. In the same way, the rate of leaf victimization corresponds to the rate of infection of healthy sites located on healthy leaves, within plants that can be healthy (VSHP sites) or infected (VSIP sites). The rate of leaf victimization thus equals $\operatorname{rinf}_{\mathrm{HP}}(t)+\operatorname{rinf}_{\mathrm{IP}}(t)$.

Sensitivity analysis. A sensitivity analysis was performed to assess the effects of allo-deposition and host structure on disease epidemics. Four patterns of allo-deposition were considered. A first pattern corresponded to random dispersion of propagules over the crop canopy. For random dispersion, the autodeposition ratio was set as the number of sites per leaf divided by the total number of sites, the allo-leaf-deposition ratio was set as the number of sites per plant minus the number of sites per leaf divided by the total number of sites, and the allo-plant-deposition ratio was set as 1 minus the sum of the autodeposition and allo-leafdeposition ratios. The allo-plant-deposition ratio also equals 1 minus 1 over the total number of plants. In the second pattern of allo-deposition, the autodeposition ratio equaled 0.34 , and the two other ratios equaled 0.33 . The third pattern corresponded to relatively high autodeposition (0.75), associated with allo-leaf- and allo-plant-deposition ratios of 0.2 and 0.05 , respectively. In the last pattern, very strong autodeposition was considered, with ratios of auto-, allo-leaf-, and allo-plant-deposition set to $0.89,0.1$, and 0.01 , respectively. For each of the four patterns of allo-deposition, two structures of canopy were considered. The first host structure was defined with 100 sites per leaf and 10 leaves per plant. The second host structure had smaller leaves (50 sites per leaf) and a larger number of leaves per plant (20). In all simulations, the following parameters were kept constant: the total number of sites was $10^{6}$, the latent period duration was 5 days, the infectious period was 10 days, and dmfr was $0.25 \mathrm{day}^{-1}$. All simulated epidemics were initiated with 10 lesions located on 10 different plants in a total population of 1,000 plants.

Simulated outputs were represented in several ways for all eight simulations (four patterns of allo-deposition and two types of host structure). First, the dynamics of the diseased host units (site, leaf, and plant) were displayed as plots over time. Second, linear regressions of the logit of the proportion of simulated diseased units over time were calculated, and slope coefficients (apparent infection rates) (30) were computed. Third, the effects of allo-deposition and host structure on spatial structure of simulated epidemics were displayed by plotting the proportion of diseased entities at higher scales versus the proportion of diseased entities at lower scales. For a given proportion of infected sites, a high proportion of diseased leaves indicates that infected sites are located on a large number of leaves, whereas a low proportion of diseased leaves indicates that infected sites are restricted to a lower number of leaves, and thus that disease is more aggregated. The same reasoning applies to leaf and plant scales. In general, when the ratio of disease incidence on a plant scale to disease incidence on a leaf scale is plotted over the course of an epidemic, the resulting curves with steeper slopes indicate epidemics with a low level of aggregation, whereas curves with flatter slopes indicate stronger aggregation. The same applies when ratios of disease incidence on a leaf scale to proportion of infected sites are plotted.

\section{RESULTS}

The model was evaluated by assessing the behavior of the system and the dynamics of the different state variables and rates of change of variable. The sigmoid shape of incidence curves at the different scales conformed with the expected behavior of the model. Changes in the parameters led to changes in simulated epidemics that also agreed with expectations (data not shown).
Epidemics were slower when $\mathrm{dmfr}$ and the infectious period were decreased and when the latency period was increased $(30,34,38)$.

The patterns of allo-deposition and host structure greatly affected simulated epidemics (Fig. 2). Epidemic development at the three scales (site, leaf, and plant) was slower when autodeposition was increased (Fig. 2A to D and E to H). For example, in the first type of host structure considered (100 sites per leaf, 10 leaves per plant), disease incidence at the plant scale reached 1 at days 74 , 86 , and 111 when simulations were performed from random dissemination to relatively high autoinfection (Fig. $2 \mathrm{~A}$ to C). In contrast, plant incidence was only 0.89 at day 120 in the case of very strong autodeposition (Fig. 2D). At low levels of autodeposition (Fig. 2A, B, E, and F), disease epidemics at the plant scale were similar for both host structures. When considering higher levels of autodeposition (Fig. 2C, D, G, and H), disease epidemics at the plant scale progressed more slowly in host structures with small leaves (50 sites per leaf, Fig. $2 \mathrm{G}$ and $\mathrm{H}$ ) than in host structures with larger leaves (100 sites per leaf, Fig. 2C and D). The same trend was observed when comparing epidemics between the two host structures at the site scale; there was no difference at low level of autodeposition, and epidemics progressed more slowly in host structures with small leaves than in host structures with large leaves at high levels of autodeposition. The epidemics at the leaf scale were delayed in host structures with large leaves (Fig. 2E to H) compared with that of host structures with smaller leaves (Fig. $2 \mathrm{~A}$ to $\mathrm{D})$.

Coefficients of determination were above 0.98 in all eight regressions of the logit of the proportion of simulated diseased entities over time. The apparent rates of infection (i.e., slope coefficients) ranged from 0.04 to $0.11 \mathrm{day}^{-1}$ and decreased when autodeposition was increased (Table 2). At high levels of autodeposition ( 0.75 or 0.89 ; last two lines of Table 2 ), the apparent rate of infection at the site scale was larger in host structures with large leaves than in host structures with small leaves, whereas they were similar at lower levels of autodeposition. The same pattern was observed when comparing the apparent rates of infection at the leaf and plant scales. When comparing the apparent rates of infection between scales (plant, leaf, and site) within each host structure, the ranking depended on the pattern of allo-deposition. When random dissemination of propagules was simulated (Table 2, line 1), the apparent rate of infection was highest at the leaf and plant scale and lowest at the site scale. When the auto-, allo-leaf-, and allo-plant-deposition ratios were $0.34,0.33$, and 0.33 , respectively, the apparent rate of infection was highest at the leaf scale and lowest at the plant and site scale. When the autodeposition ratio was high ( 0.75 or 0.89 ; last two lines of Table 2$)$, the apparent rate of infection was highest at the site scale, lowest at the plant scale, and intermediate at the leaf scale.

The slopes of curves representing the proportion of diseased leaves as a function of the proportion of infected sites decreased when the autodeposition ratio was increased (Fig. 3A). In the same way, when considering the leaf and plant scales, the slopes of curves decreased when autodeposition was increased (Fig. 3B). These results indicate that disease aggregation increased when autodeposition was increased. In a given pattern of allo-deposition, the slope of the curve relating leaf and site scales was always higher in the host structure with large leaves (100 sites per leaf, 10 leaves per plant) compared with that of the host structure with smaller leaves (50 sites per leaf, 20 leaves per plant) (Fig. 3A). The opposite ranking was observed when considering plant and leaf scales (Fig. 3B).

\section{DISCUSSION}

Model evaluation. The model outputs in the case of random dispersal of the propagules were compared with results expected from two other approaches. First, the apparent rate of infection 
100 sites/leaf

10 leaves/plant
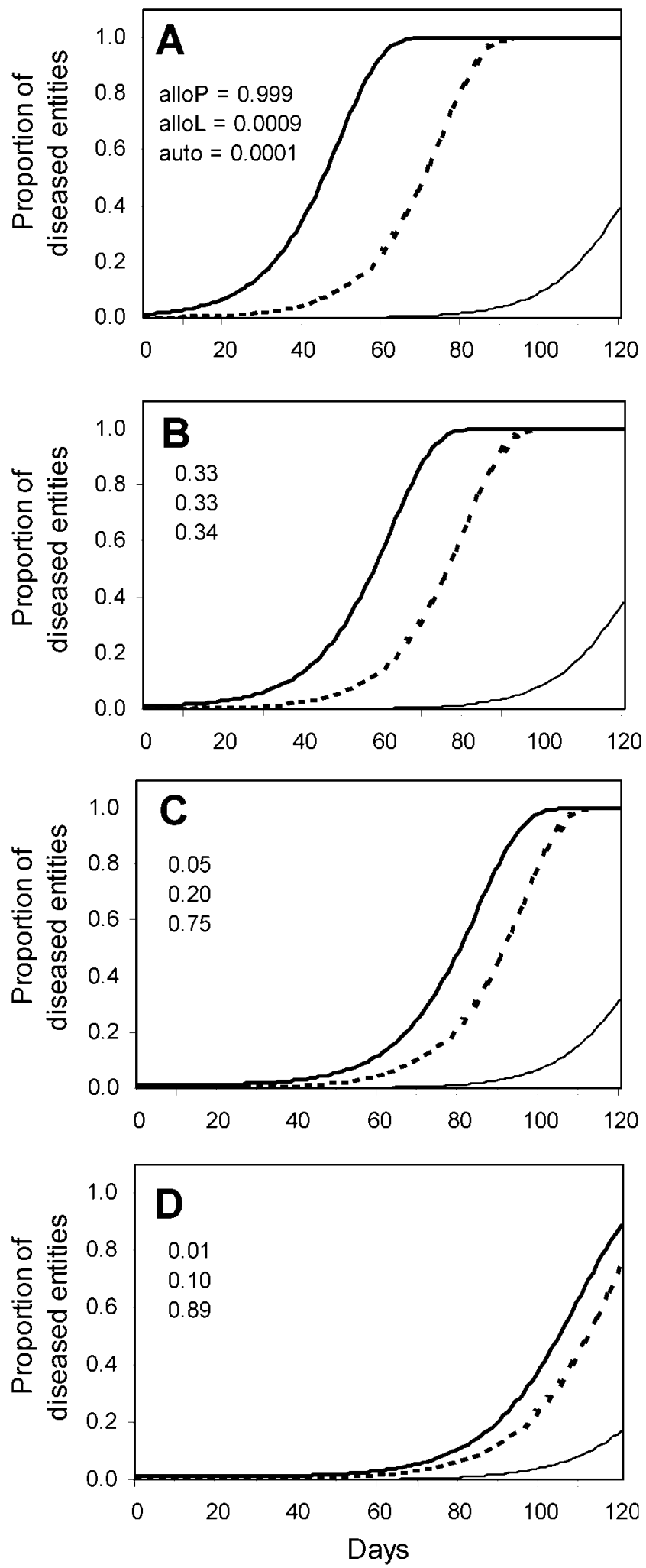

50 sites/leaf 20 leaves/plant
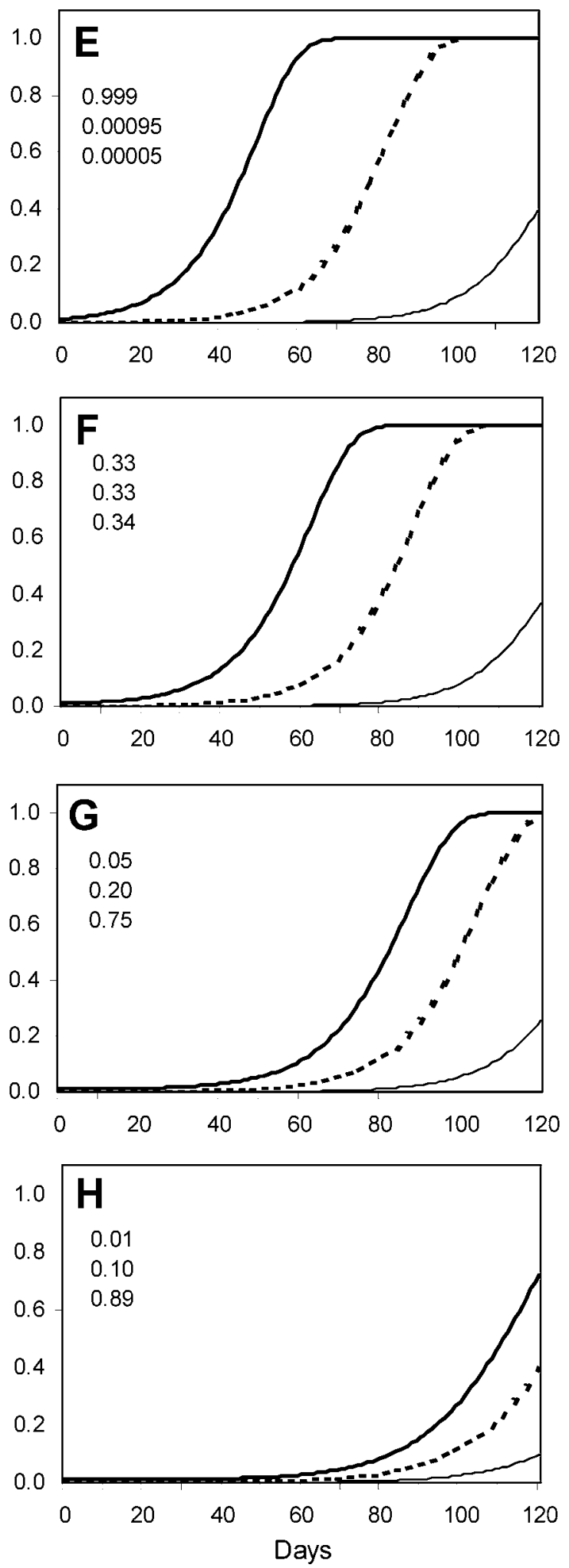

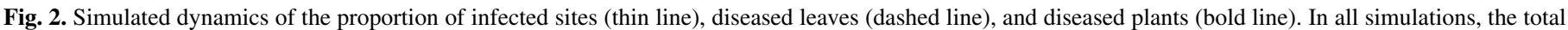

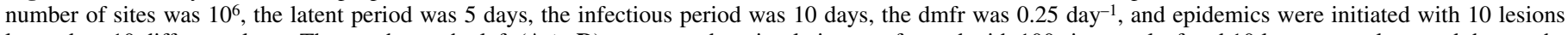

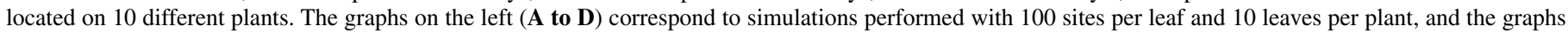

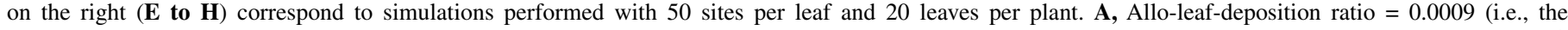

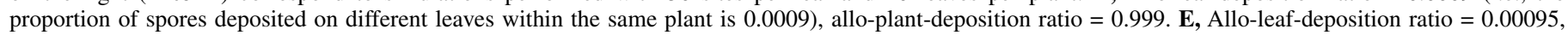

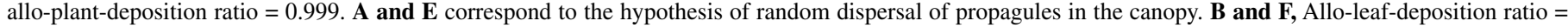

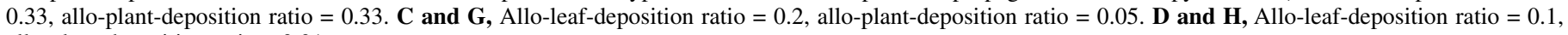
allo-plant-deposition ratio $=0.01$. 
was estimated from regression of the logit of proportion of infected sites over time in the case of a simpler structure, which considers the site scale only (the model designed by Zadoks [38]). The apparent rate of infection was 0.0906, which is equal to the value estimated from our simulations under the hypothesis of random dissemination of propagules. The model used in this study therefore performs identically to that of Zadoks (38), in regards to random propagule dispersal. Second, the relationship between disease incidence at higher and lower scales obtained from simulations under the hypothesis of random dispersal of propagules (Fig. 3A and B) was compared with the theoretical relationship obtained when considering that the frequency distribution of diseased entities at the lower scale is a binomial distribution. Under this hypothesis, which reflects a random distribution of disease, the relationship can be written as $(8,13)$ :

$$
I_{\text {high }}=1-\left(1-I_{\text {low }}\right)^{n}
$$

where $I_{\text {high }}$ is the incidence at higher level of integration, $I_{\text {low }}$ is the incidence at lower level of integration, and $n$ is the number of entities at the lower scale per entity at higher scale. This relationship can be established in two cases: between plant and leaf scales and between leaf and site scales. The differences between simulated and theoretical values were smaller than $1.5 \%$ in both cases, indicating that the model outputs are in agreement with the expected (theoretical) values (equation 12), under the hypothesis of random distribution of the disease.

Model hypothesis for infection from autodeposition. In equation 6 , infection from autodeposition is written as the product, number of infectious sites (INFSI) $\times \mathrm{dmfr} \times$ ratio of autodeposition $\times \operatorname{cofr}_{\mathrm{IL}}$. Autodeposition is thus modeled as if the propagules dispersed from all diseased leaves in a canopy were pooled together and then homogeneously deposited on diseased leaves. This is a simplification of the autodeposition process that occurs in reality, where propagules from each leaf are dispersed and then deposited on the same leaf. The population of leaves carrying infectious sites may include leaves with varying numbers of infectious sites (lesions). In that case, a large proportion of the dispersed propagules may originate from leaves carrying a large number of infectious sites (compared with that of leaves carrying few infectious sites) and be deposited on leaves with a small conditional severity. In the model, cofr $_{\mathrm{IL}}$ is defined as 1 minus the conditional severity, and is computed over all diseased leaves, the newly infected ones (which have fewer lesions and, therefore, produce fewer propagules) and those that have been infected before (and where disease intensification has taken place). Equation 6 may thus overestimate infection through autoinfection.

We estimated, numerically, the product INFSI by $\operatorname{cofr}_{\text {IL }}$ under the hypothesis of autodeposition of propagules on the leaf from which they were dispersed. This product was noted as INCOmod:

$$
\operatorname{INCOmod}=\sum_{i=1}^{n}\left[\operatorname{INFSI}_{i}(t) \times \operatorname{COFRIL}_{i}(t)\right] \text { with } \sum_{i=1}^{n}\left[\operatorname{INFSI}_{i}(t)\right]=\operatorname{INFSI}(t)
$$

where $n$ is the number of infectious lesions per leaf, $\operatorname{INFSI}_{i}$ is the number of infectious sites present on leaves with $i$ infectious sites per leaf, and COFRIL $\mathrm{C}_{i}$ is the proportion of vacant sites present on leaves with $i$ infectious sites per leaf. If latent and removed sites are not accounted for, $\mathrm{COFRIL}_{i}$ can be approximated as 1 (i/nsiperLf). INFSI $_{i}$ can be written as $i \times \operatorname{DISLF}_{i}$, where $\operatorname{DISLF}_{i}$ is the number of diseased leaves with $i$ infectious sites per leaf. DISLF $_{i}$ follows a binomial distribution if the disease is randomly distributed. If disease is aggregated, DISLF $_{i}$ can be assumed to follow the beta-binomial distribution $(7,9)$. INCOmod was calculated for varying values of $p$ (the mean proportion of infectious sites per leaf) in two cases: $N=30$ sites per leaf and $N=100$ sites per leaf. INCOmod was first computed with DISLF $_{i}$ following a binomial distribution, and second for $\mathrm{DISLF}_{i}$ following a betabinomial distribution, for different values of the parameter theta of the beta-binomial distribution.

We then computed the product INFSI by cofr $\mathrm{IL}_{\mathrm{IL}}$ as in the model. This product was noted as INCO, with $\operatorname{INCO}=\operatorname{INFSI}(t) \times[1-$ $\operatorname{cincS}(t)]$. The ratio INCOmod/INCO was then computed. This ratio did not vary with $p$. It was 0.99 in the case of DISLF $_{i}$ following a binomial distribution. It varied greatly with theta when computed for DISLF $i$ following a beta-binomial distribution. The relationship between the ratio INCOmod/INCO and theta is displayed in Figure 4 for theta values varying between 0 and 3. This range was determined from reported values of theta in several pathosystems. Maximum theta values were 0.07 to 0.17 when aggregation of diseased plants was assessed $(7,11,23,33) ; 0.4$ to 1 when aggregation of diseased leaflets or leaves was evaluated $(10,24,25)$; and 3 when disease aggregation of tillers within plants was assessed (19). The ratio INCOmod/INCO was virtually not affected by $N$ (Fig. 4). The ratio was 1 when theta equaled 0 , and declined to 0.24 when theta was 3 (very high aggregation). One possible improvement of the model could be to use the curve displayed in Figure 4 to introduce a new parameter that would correct equation 6 according to aggregation. The relationships between theta and the other model variables remain to be established. One conclusion of this finding is that autodeposition may reduce the rate of epidemic progress much more than that simulated by the model.

Effects of allo-deposition patterns and host structure on epidemics. Epidemics were slower when allo-leaf- and allo-plantdeposition ratios were small (Fig. 2; Table 2). This effect was more important when considering the leaf and plant scales than when considering the site scale. The decrease in allo-deposition ratios indeed affects the number of propagules deposited on different leaves and plants, and thus decreases the apparent rate of infection of leaves and plants. On the other hand, the increase in autodeposition causes faster disease intensification on diseased leaves, thus locally reducing the number of available sites, and so reducing the rate of incidence increase at the site scale. When allo-deposition ratios were decreased, the apparent rate of infection decreased more in the case of small leaves than in the case of

TABLE 2. Apparent rate of infection in the simulations performed with four patterns of allo-deposition, and two host structures ${ }^{\mathrm{a}}$

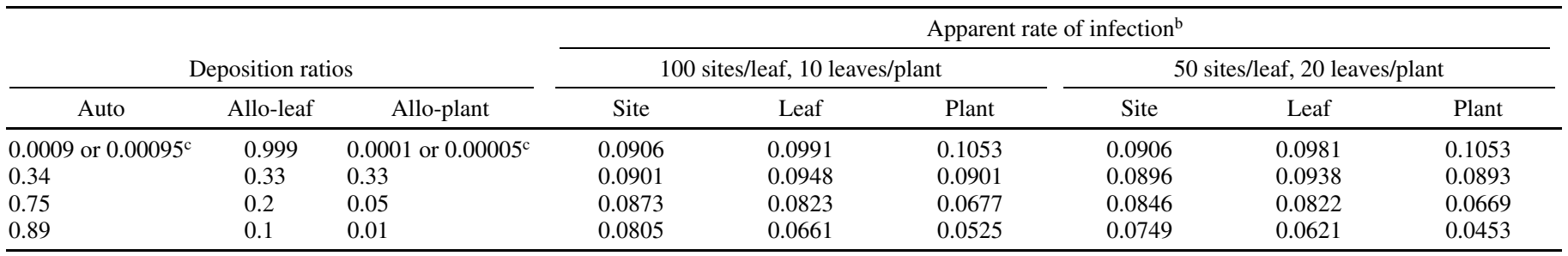

a In all simulations, the total number of sites was $10^{6}$, the latent period was 5 days, the infectious period was 10 days, the dmfr was 0.25 day ${ }^{-1}$, and epidemics were initiated with 10 lesions located on 10 different plants.

${ }^{\mathrm{b}}$ The apparent rate of infection is the slope parameter determined from a linear regression of the logit of the proportion of diseased entities (site, leaf, or plant) over time.

${ }^{\mathrm{c}}$ The first number is the parameter used in the simulation with 100 sites per leaf and 10 leaves per plant, and the second number is used in the simulation with 50 sites per leaf and 20 leaves per plant. These parameters correspond to a random dispersal of the propagules in the canopy. 
large leaves. This result indicates that there is an interaction between the effects of host structure and pattern of dispersal of propagules on the speed of epidemics; on the one hand, the speed of epidemics is more affected by high autodeposition when host
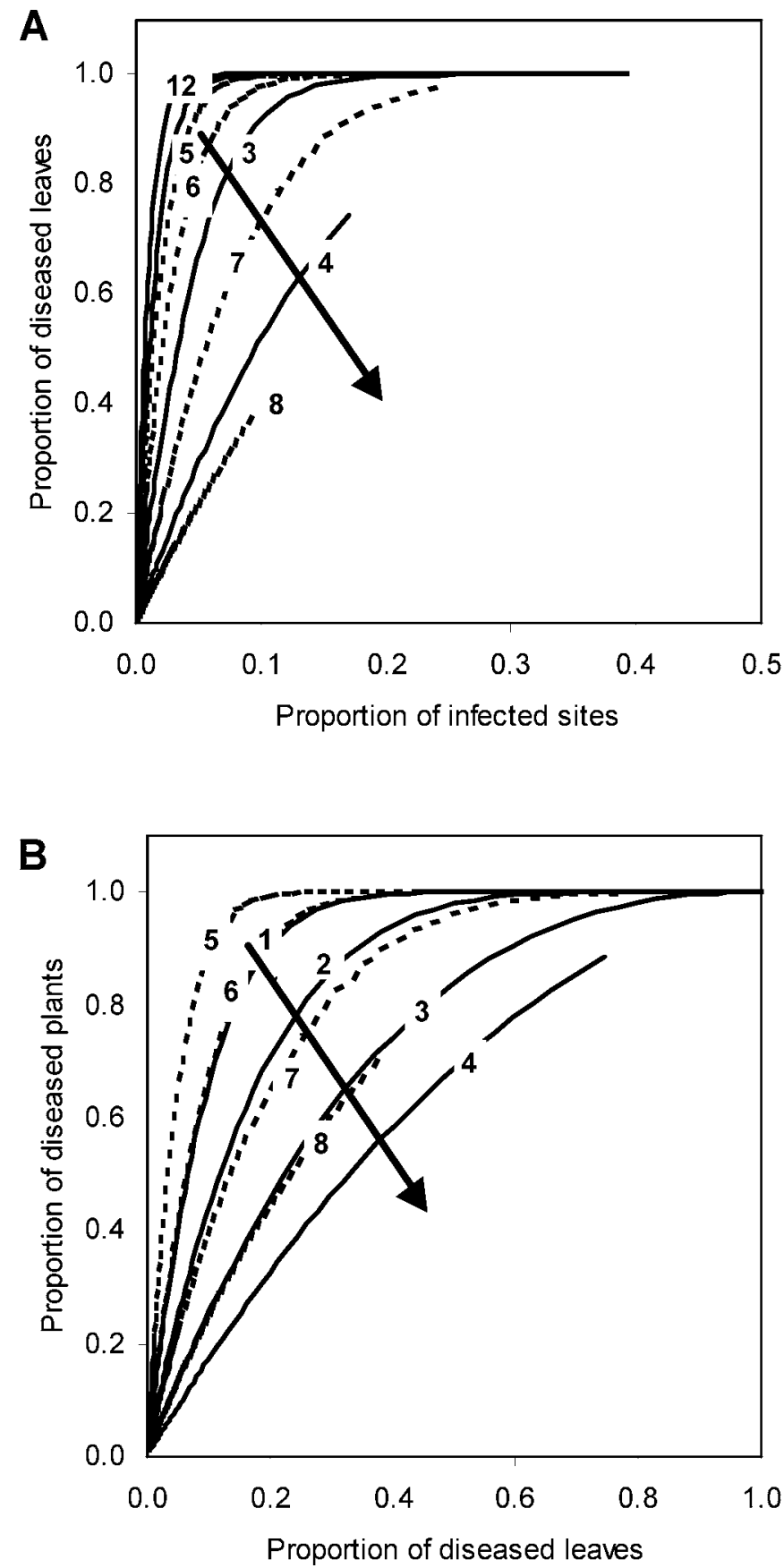

Fig. 3. Relationships between simulated disease incidence at higher scale and disease incidence at lower scale. A, Proportion of diseased leaves as a function of proportion of infected sites. B, Proportion of diseased plants as a function of proportion of diseased leaves. In all simulations, the total number of sites was $10^{6}$, the latent period was 5 days, the infectious period was 10 days, the dmfr was $0.25 \mathrm{day}^{-1}$, and epidemics were initiated with 10 lesions located on 10 different plants. Curves 1 to 4 , simulations performed with 100 sites per leaf and 10 leaves per plant; curves 5 to 8 , simulations performed with 50 sites per leaf and 20 leaves per plant. Curve 1: allo-leaf-deposition ratio $=$ 0.0009, allo-plant-deposition ratio $=0.999$. Curve 5: allo-leaf-deposition ratio $=0.00095$, allo-plant-deposition ratio $=0.999$. Curves 1 and 5 correspond to the hypothesis of random dispersal of propagules in the canopy. Curves 2 and 6: allo-leaf-deposition ratio $=0.33$, allo-plant-deposition ratio $=$ 0.33. Curves 3 and 7: allo-leaf-deposition ratio $=0.2$, allo-plant-deposition ratio $=0.05$. Curves 4 and 8 : allo-leaf-deposition ratio $=0.1$, allo-plant-deposition ratio $=0.01$. Arrows on the graphs indicate increasing autodeposition. structures involve small leaves than when they involve larger leaves. This can be attributed again to the fewer number of sites locally available for infection in the case of smaller leaves. On the other hand, the speed of epidemics is only slightly affected by host structure at low autodeposition ratios. In this case, local dispersal is low, and the effect of leaf size on the number of available sites is reduced.

The epidemic at the leaf scale was delayed in the case of smaller leaves (50 sites per leaf) compared with that of larger leaves (100 sites per leaf). There were twice as many leaves in the simulations involving small leaves than in simulations performed with large leaves, but the number of initial lesions was the same in all simulations. Thus, the delay is simply due to the fact that primary inoculum in terms of proportion of diseased leaves is reduced by half in simulations performed with small leaves.

The second host structure addressed in the sensitivity analysis (50 sites per leaf) was interpreted as a case with smaller leaves. This could also be interpreted as a situation in which the size of a lesion is twice that of the first host structure considered (100 sites per leaf). This would then suggest that epidemics involving large lesions are more affected by increases in autodeposition than epidemics involving smaller lesions.

The relationships between disease incidence at a larger scale and disease incidence at a lower scale (Fig. 3) indicate that the slope of the curves increased as the ratios of allo-leaf- and alloplant-deposition were increased. This trend agrees with several studies $(8,13,14)$ that showed that the slope of the curve increases when disease aggregation decreases.

Estimation of auto- and allo-deposition ratios. Auto- and allo-deposition ratios are key parameters in the model, and they indeed are important biological parameters for the spatiotemporal development of epidemics. alloP is also one parameter to consider when assessing the efficiency of cultivar mixture to control epidemics (17). These parameters have nevertheless been measured in a few instances only (brown rust of wheat [18]; angular leaf spot of bean [D. Allorent, L. Willocquet, J. L. Janneau, and S. Savary, unpublished data]). This may be explained by the difficulties with experimental measurement of these parameters.

The model presented here provides a mathematical framework to establish relationships between ratios of auto- and allo-deposi-

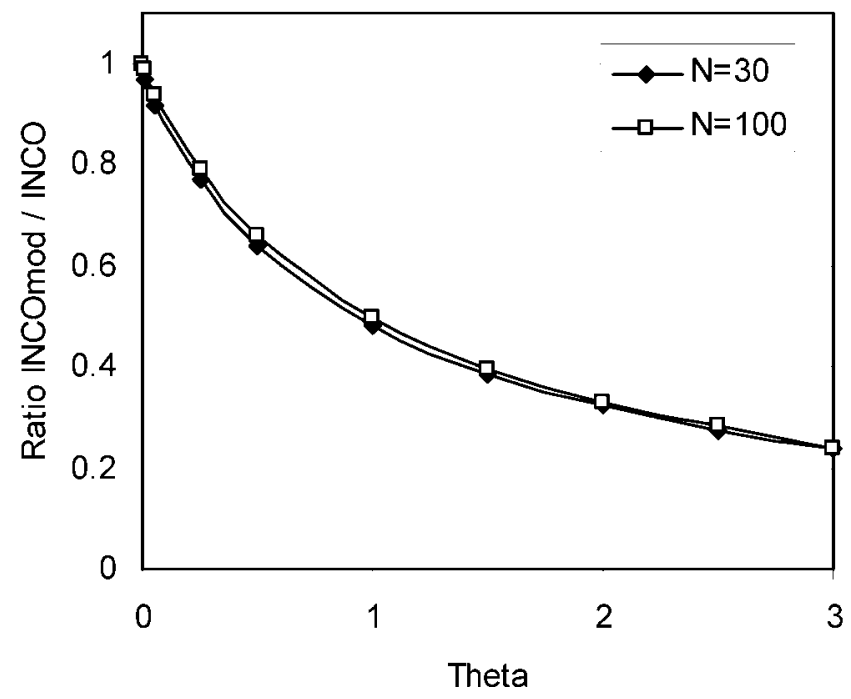

Fig. 4. Relationship between the ratio INCOmod/INCO and the parameter theta of the beta-binomial distribution. $N$, number of sites per leaf. INCOmod is the product of the number of infectious sites by $\operatorname{cofr}_{I L}\left(\operatorname{cofr}_{I L}\right.$ is the COFR for diseased leaves), computed under the hypothesis of autodeposition of propagules on the leaf from which they were dispersed. INCO is also the product of the number of infectious sites by cofr $_{I L}$, but computed under the hypothesis of the model, where spores involved in autodeposition are dispersed and then pooled and redistributed homogeneously over the infected leaves. 
tion and disease incidences at the three scales. The curves obtained in Figure $3 \mathrm{~B}$ correspond to relationships that can be derived from equations 7 and 8 :

$$
\begin{gathered}
\frac{\Delta \text { DISPL }}{\Delta \text { DISLF }}=\frac{\operatorname{rinf}_{\mathrm{HP}}}{\operatorname{rinf}_{\mathrm{HP}}+\operatorname{rinf}_{\mathrm{IP}}}= \\
\text { alloP } \times\left(1-\text { incP }^{2}\right.
\end{gathered}
$$$$
\overline{[\text { alloP } \times(1-\operatorname{incP})]+[(\text { alloP } \times \operatorname{inc} \mathrm{P} \times(1-\operatorname{cincL}))+(\text { alloL } \times(1-\operatorname{cincL}))]}
$$

This reduces to the following equation when disease incidence is small:

$$
\frac{\Delta \mathrm{DISPL}}{\Delta \mathrm{DISLF}} \approx \frac{\text { alloP }}{\text { alloP }+ \text { alloL }}
$$

or

$$
\frac{\Delta \text { incP }}{\Delta \text { incL }} \approx\left(\frac{\text { alloP }}{\text { alloL }+ \text { alloP }}\right) \times \text { nlfperPl }
$$

In the same way, using equations 6,7 , and 8 , the curves obtained in Figure 3A correspond to the following equation when disease incidence is small:

$$
\frac{\Delta \text { DISLF }}{\Delta \text { INSI }} \approx \text { alloL }+ \text { alloP }
$$

or

$$
\frac{\Delta \text { incL }}{\Delta \text { incS }} \approx(\text { alloL }+ \text { alloP }) \times \text { nsiperLf }
$$

where INSI is the number of infected sites (number of lesions).

Equations 14 and 16 allow to derive alloP into a very simple expression:

$$
\text { alloP } \approx \frac{\Delta \text { DISPL }}{\Delta \text { INSI }}
$$

or, using equations 15 and 17 ,

$$
\text { alloP } \approx \frac{\Delta \text { incP }}{\Delta \text { incS }} / \text { nsiperPl }
$$

Equations 18 and 19 and 16 and 17 might then provide a means to estimate alloP and alloL + alloP (that is, 1 - auto), respectively, when disease incidence is small.

Underlying hypotheses of the model. The estimates of the different ratios of auto- and allo-deposition described previously (equations 16 to 19) correspond to low disease intensity, i.e., early stages of epidemics, and to specific assumptions of the model. Departures of epidemiological systems from these assumptions have to be inspected carefully in order to assess when equations 16 to 19 can be used. We briefly address five of these assumptions below. A necessary condition to estimate these ratios from field data is that the variation in the number of lesions measured in the beginning of the epidemic should be mainly due to secondary infections (i.e., no significant number of lesions originating from primary inoculum). Another important condition is that new lesions should originate from propagules produced in the system's area (i.e., no significant amount of incoming inoculum from areas outside the considered field or plot). Furthermore, the multiple infection transformation (6) should be used if necessary (i.e., if several propagules dispersed away from a diseased leaf may infect simultaneously the same healthy leaf). Gregory's multiple infection transformation (6) actually was incorporated in the model, but this did not significantly alter the behavior of the model with the parameters used here and a daily time-step (data not shown). In order to keep the model simple, Gregory's transformation was not incorporated in the equations. Changes in parameters, and certainly, in time-step, would render Gregory's transformation necessary.

Host growth through leaf expansion may not alter the relevance of the estimates, but host growth through emergence of new leaves may lead to underestimation of the allo-leaf-deposition ratio, because new, healthy leaves may appear between dispersal of propagules and lesion emergence. Finally, for epidemics where rapid lesion expansion takes place, the autodeposition ratio may be underestimated, because healthy tissue availability for autoinfection would be decreased by the increase in lesion size. Also, lesion expansion corresponds to a process different from infection, but nevertheless contributes to increasing the number of occupied sites and to what commonly is measured as severity. When present, lesion expansion should therefore be accounted for in both the model and the field data used for model evaluation.

Potential applications of the model. The structure of the model provides new insight into the relationships between disease incidence at different levels of hierarchy in a plant population, with parameters that have a biological meaning. It provides a way to estimate ratios of allo-deposition within the limits given in the preceding paragraph. It could thus help to characterize pathosystems in relation to the spatiotemporal epidemics they can generate. In addition, the model can provide a basis for assessing the potential efficiency of mixtures of resistant and susceptible cultivars in a given pathosystem (17). Finally, the model provides a means to investigate the effects of the interaction between host architecture and pathogen dissemination on epidemic dynamics.

Generic value of the model. The scales addressed by the model are site, leaf, and plant, in a crop canopy. Other organization scales could be considered with this model, such as plant, field, and region, in an agroecosystem. The model presented does not include specific characteristics of pathosystems and could represent epidemics involving airborne, as well as soilborne, propagules. This model may represent ecological processes that occur in fields of study other than phytopathology, where migration at different spatial scales would be considered $(1,3)$.

\section{ACKNOWLEDGMENTS}

We thank the anonymous reviewers for their helpful suggestions to improve the manuscript.

\section{LITERATURE CITED}

1. Amarasekare, P., and Possingham, H. 2001. Patch dynamics and metapopulation theory: The case of successional species. J. Theor. Biol. 209:333-344.

2. Bainbridge, A., and Stedman, O. J. 1979. Dispersal of Erysiphe graminis and Lycopodium clavatum spores near to the source in a barley crop. Ann. Appl. Biol. 91:187-198.

3. Bascompte, J. 2001. Aggregate statistical measures and metapopulation dynamics. J. Theor. Biol. 209:373-379.

4. Ferrandino, F. J. 1993. Dispersive epidemic waves: I. Focus expansion within a linear planting. Phytopathology 83:795-802.

5. Fitt, B. D. L., and McCartney, H. A. 1986. Spore dispersal in relation to epidemic models. Pages 311-345 in: Plant Disease Epidemiology, vol. 1. Population Dynamics and Management. K. J. Leonard and W. E. Fry, eds. Macmillan Publishing Company, New York.

6. Gregory, P. H. 1948. The multiple-infection transformation. Ann. Appl. Biol. 35:412-417.

7. Hughes, G., and Madden, L. V. 1993. Using the beta-binomial distribution to describe aggregated patterns of disease incidence. Phytopathology 83:759-763.

8. Hughes, G., McRoberts, N., Madden, L. V., and Gottwald, T. R. 1997. Relationships between disease incidence at two levels in a spatial hierarchy. Phytopathology 87:542-550.

9. Madden, L. V., and Hughes, G. 1995. Plant disease incidence: Heterogeneity, and temporal analysis. Annu. Rev. Phytopathol. 33:529-564.

10. Madden, L. V., Hughes, G., and Ellis, M. A. 1995. Spatial heterogeneity of the incidence of grape downy mildew. Phytopathology 85:269-275.

11. Madden, L. V., Nault, L. R., Murral, D. J., and Apelt, M. R. 1995. Spatial pattern analysis of the incidence of aster yellows disease in lettuce. Res. Pop. Ecol. 37:279-289.

12. McCartney, H. A., and Bainbridge, A. 1984. Deposition gradients near to a point source in a barley crop. Phytopathol. Z. 109:219-236.

13. McRoberts, N., Hughes, G., and Madden, L. V. 1996. Incorporating spatial variability into simple disease progress models for crop pathogens. 
Aspects Appl. Biol. 46:75-82.

14. McRoberts, N., Hughes, G., and Madden, L. V. 2003. The theoretical basis and practical application of relationships between different disease intensity measurements in plants. Ann. Appl. Biol. 142:191-211.

15. Minogue, K. P., and Fry, W. E. 1983. Models for the spread of disease: Model description. Phytopathology 73:1168-1173.

16. Minogue, K. P., and Fry, W. E. 1983. Models for the spread of plant disease: Some experimental results. Phytopathology 73:1173-1176.

17. Mundt, C. C., and Leonard, K. J. 1986. Analysis of factors affecting disease increase and spread in mixtures of immune and susceptible plants in computer-simulated epidemics. Phytopathology 76:832-840.

18. Robert, C. 2003. Etude et modélisation du fonctionnement d'un couvert de blé attaqué par le complexe parasitaire Puccinia triticina-Mycosphaerella graminicola. Ph.D. thesis. INA-PG, France.

19. Savary, S., Castilla, N., and Willocquet, L. 2001. Analysis of the spatiotemporal structure of rice sheath blight epidemics in a farmer's field. Plant Pathol. 50:53-68.

20. Savary, S., and Van Santen, G. 1992. Effect of crop age on primary gradients of late leaf spot (Cercosporidium personatum) on groundnut. Plant Pathol. 41:265-273.

21. Savary, S., Willocquet, L., and Teng, P. S. 1997. Modelling sheath blight epidemics on rice tillers. Agric. Syst. 55:359-384.

22. Scherm, A. 1996. On the velocity of epidemic waves in model plant disease epidemics. Ecol. Mod. 87:217-222.

23. Tanne, E., Marcus, R., Dubitzky, E., and Raccah, B. 1996. Analysis of progress and spatial pattern of corky bark in grapes. Plant Dis. 80:34-38.

24. Turechek, W. W., and Madden, L. V. 1999. Spatial pattern analysis of strawberry leaf blight in perennial production systems. Phytopathology 89:421-433.

25. Turechek, W. W., and Madden, L. V. 1999. Spatial pattern analysis and sequential sampling for the incidence of leaf spot on strawberry in Ohio. Phytopathology 89:992-1000.

26. Turechek, W. W., and Madden, L. V. 2001. Effect of scale on plant disease incidence and heterogeneity in a spatial hierarchy. Ecol. Mod. 144:77-95.

27. Turechek, W. W., and Madden, L. V. 2003. A generalized linear modeling approach for characterizing disease incidence in a spatial hierarchy.
Phytopathology 93:458-466.

28. van den Bosch, F., Zadoks, J. C., and Metz, A. J. 1988. Focus expansion in plant disease. I. The constant rate of focus expansion. Phytopathology 78:54-58.

29. van den Bosch, F., Zadoks, J. C., and Metz, A. J. 1988. Focus expansion in plant disease. II. Realistic parameter-sparse models. Phytopathology 78:59-64.

30. Van der Plank, J. E. 1963. Plant Diseases: Epidemics and Control. Academic Press, New York.

31. Waggoner, P. E., and Rich, S. 1981. Lesion distribution, multiple infection, and the logistic increase of plant disease. Proc. Natl. Acad. Sci. USA 78:3292-3295.

32. Willocquet, L., Fernandez, L., and Savary, S. 2000. Effect of various crop establishment methods practiced by Asian farmers on epidemics of rice sheath blight, caused by Rhizoctonia solani. Plant Pathol. 49:346-354.

33. Xiao, C. L., Hao, J. J., and Subbarao, K. V. 1997. Spatial patterns of microsclerotia of Verticillium dahliae in soil and Verticillium wilt of cauliflower. Phytopathology 87:325-331.

34. Xu, X. M., and Ridout, M. S. 1998. Effects of initial epidemic conditions, sporulation rate, and spore dispersal gradient on the spatio-temporal dynamics of plant disease epidemics. Phytopathology 88:1000-1012.

35. Yang, X. B., Berggren, G. T., and Snow, J. P. 1990. Effect of free moisture and soybean growth stage on focus expansion of Rhizoctonia aerial blight. Phytopathology 80:497-503.

36. Yang, X. B., Snow, J. P., and Berggren, G. T. 1991. Patterns of Rhizoctonia foliar blight on soybean and effect of aggregation on disease development. Phytopathology 81:287-293.

37. Yang, X. B., and TeBeest, D. O. 1992. Dynamic pathogen distribution and logistic increase of plant disease. Phytopathology 82:380-383.

38. Zadoks, J. C. 1971. Systems analysis and the dynamics of epidemics. Phytopathology 61:600-610.

39. Zadoks, J. C., and Kampmeijer, P. 1977. The role of crop populations and their deployment, illustrated by means of a simulator EPIMUL 76. Ann. N.Y. Acad. Sci. 287:164-190.

40. Zawolek, M. W., and Zadoks, J. C. 1992. Studies in focus development: An optimum for the dual dispersal of pathogens. Phytopathology 82: 1288-1297. 